\title{
Genetic diversity of grapevine (Vitis vinifera L.) as revealed by ISSR markers
}

\author{
Rezq Basheer-Salimia • Arwa Mujahed
}

Received: 1 February 2019 / Revised: 15 February 2019 / Accepted: 15 February 2019

(c) Korean Society for Plant Biotechnology

\begin{abstract}
The main goal of this study was to determine the genetic diversity among 36 grape cultivars grown in Palestine by using ISSR-polymerase chain reaction (PCR) fingerprints. Among the tested primers, 17 produced reasonable amplification products with high intensity and pattern stability.

A total of 57 DNA fragments (loci) separated by electrophoresis on agarose gels were detected and they ranged in size, from 150 to $900 \mathrm{bp}$. Out of these fragments, 55 (88\%) were polymorphic and $2(3.5 \%)$ monomorphic. Our results also revealed an average of 3.1 loci per primer. A minimum of 1 and maximum of 10 DNA fragments were obtained (S-17, \#820 and \#841) and (S-31) primers, respectively. Therefore, the later primer ( $\mathrm{S}-31)$ is considered to be the most powerful primer among the tested ones.

The genetic distance matrix showed an average distance range of between 0.05 and 0.76 . The maximum genetic distance value of 0.76 (24\% similarity) was exhibited between the (Shami and Marawi.Hamadani.Adi) as well as (Bairuti and Marawi.Hamadani.Adi) genotypes. On the other hand, the lowest genetic distance of 0.05 (95\% similarity) was exhibited between (Jandali.Tawel.Mofarad and Jandali. Kurawi.Mlzlz) along with (Shami.Aswad and Shami.mtartash. mlwn) genotypes. Furthermore, the UPGMA dendrogram generally clusters the grape cultivars into eight major clusters in addition to an isolated genotype. Based on these figures, the cultivars tested in this study could be characterized by large divergence at the DNA level. This is taking the assumption that our region has a very rich and varied clonal grape genetic structure.
\end{abstract}

R. Basheer-Salimia $(\bowtie)$

Department of Plant Production and Protection, College of Agriculture, Hebron University

e-mail: rezqbasheer@hebron.edu

\section{A. Mujahed}

Department of Applied Biology, College of Applied Science, Palestine Polytechnic University
Keywords Vitis, genetic relationship, ISSR, UPGMA, JACCARD-index

\section{Introduction}

Grapevine (Vitis vinifera L.) is one of the oldest known fruit trees cultivated in Palestine mainly for wine, table fruit, juice, and raisins. Since 14 centuries (establishment of Islamic civilization), its plantation dedicated only on table grape seeded-cultivars and it was growing mainly at the southern part of West-Bank especially Hebron and Bethlehem areas (Sultan 2005), in which the geographical and environmental conditions are suitable for growing highquality fruits. Currently, it is considered as the second important fruit crop after olive in terms of areas covered, economic returns, and social values (Basheer-Salimia 2015a; MOA 2017). Lately, its plantation starts to expand into the northern regions focusing mainly on seedless grape.

The long domestication history with numerous cultivars and further introduction of new cultivars especially from the surrounding countries has resulted in ambiguity in the description and nomenclature of grape cultivars (BasheerSalimia 2015b). Furthermore, many cultivars were also subjected either to genetic deterioration and/or to disappearance leading thereby to lose a great pool of grape genetic materials. Indeed, such problems might relate to different biotic and abiotic stresses, especially the climate change which characterizes the region lately where temperature and drought is dramatically increased (Basheer-Salimia and Ward 2014). Therefore, determination of genetic variability and proper cultivar identification in grapevine would be of major importance for any future improvement programs and germplasm preservation as well.

In general, morphological (based mainly on traditional field evaluation) and/or DNA molecular markers are commonly used for detection and analyzing of genetic variation 


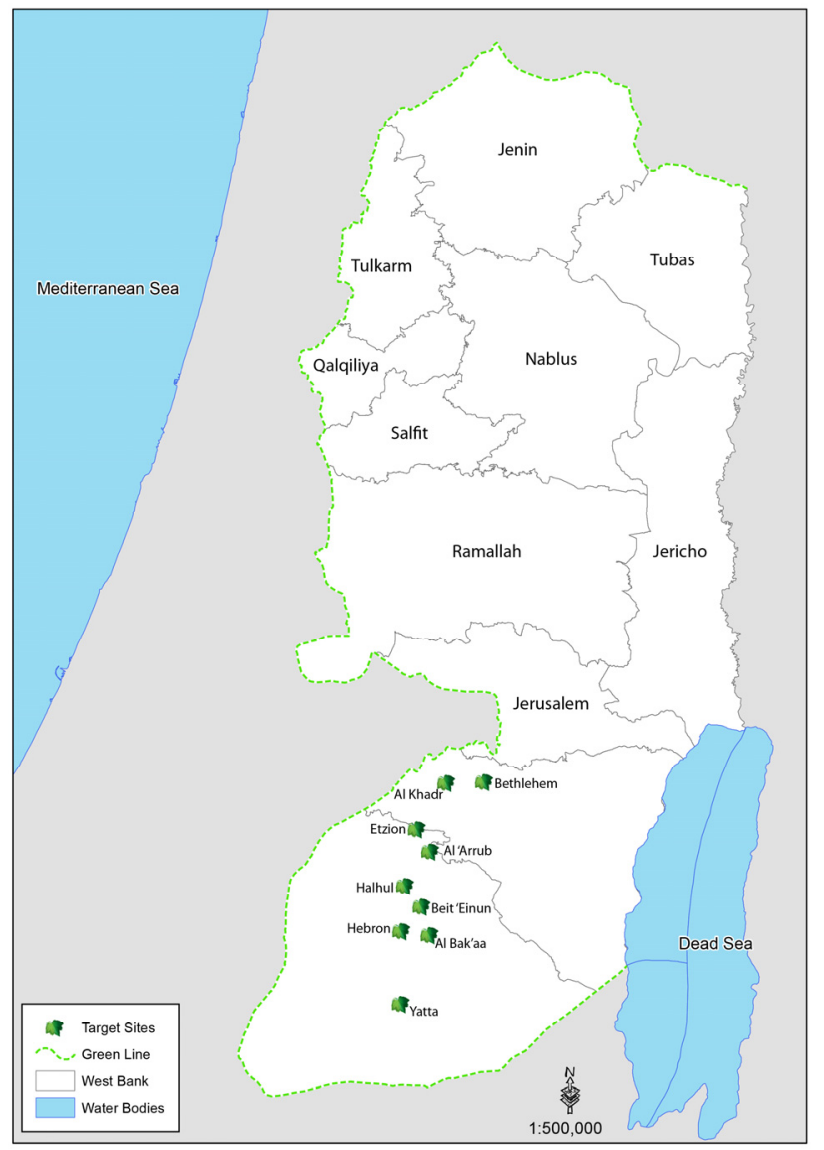

Fig. 1 Map showing the grapevine collection sites

among fruit tree species (Basheer-Salimia et al. 2012. In fact, characterizations based on morphological criteria's are considered as time-consuming, laborious and error-prone due to environmental factors, which poses great challenges (Sripholtaen et al. 2016), in addition to its high variability across years and locations (Lima et al. 2002; Achtak et al. 2009). Alternatively, DNA-based markers proved to be powerful tools to estimate genetic diversity as well as genotype identity of many horticultural species including grape (Herrera et al. 2002; Bahurupe et al. 2013). In fact, molecular markers offer numerous advantages over conventional morphological based approach, since they proved to be: (1) allow direct comparison of different genetic material independent of environmental influences (Weising et al. 1995); (2) stable and detectable in all tissues regardless of growth, differentiation, development, or defense status of the cell (Chittora et al. 2015); (3) accurate and rapid genotype identification especially in vegetatively propagated plants species (Depnath 2009), such as grape; and (4) not confounded by the environment, pleiotropic, and epistatic effects (Agarwal et al. 2008).

Comparing with the other common DNA markers; Inter Simple Sequence Repeat markers (ISSRs) have been used successfully in many fruit tree species because it's simple, fast, high stability, no prior requirement of sequence information, cost effectiveness and versatility of markers (Qian et al. 2001; Reddy et al. 2002; Choudhary et al., 2014). In grapes, numerous DNA-based markers have been conducted toward characterization of grape species in the Mediterranean region (Riaz et al. 2018); however rare studies have been found in the literature on Palestinian grapes. In the present work, we have employed ISSRs technique to determine the number of genetically different grapevine cultivars that were actually exists in Palestine; to infer possible cases of synonymy and homonymy; and to evaluate the genetic relationships of the characterized cultivars.

\section{Materials and Methods}

Plant materials

Healthy grape leaves were collected from the middle-region of the newly growing shoots from 36 assumed cultivars ( $>50$ years) throughout Hebron and Bethlehem districts (Fig. 1). 


\section{DNA extraction and purification}

Two medium - healthy, young leaves of each assumed cultivar were ground with pestle and mortar using liquid nitrogen to fine powder. A weight of $100 \mathrm{mg}$ of leaf powder of each cultivar was transferred into a $1.5 \mathrm{~mL}$ eppendrof tube and mixed with, $400 \mu \mathrm{L}$ of buffer AP1 and $4 \mu \mathrm{L}$ of RNase A stock solution $(100 \mathrm{mg} / \mathrm{mL})$ were added to the tube, and the mixture was vigorously vortexed, incubated at $65^{\circ} \mathrm{C}$, and mixed 2-3 times during incubation by inverting. A quantity of $130 \mu \mathrm{L}$ of buffer AP2 was added to the lysate, which was mixed, incubated on ice for 5 minutes, and then centrifuged at 20,000 x g. The supernatant was applied to the QIA-shredder Mini Spin Column and it was carefully centrifuged at $20,000 \times \mathrm{g}$ for 2 minutes so as not to disturb the pellet. The flow-through fraction (liquid) was transferred to a new $2 \mathrm{~mL}$ eppendrof tube without disturbing the cell-debris pellet and a quantity of 1.5 volumes of buffer AP3/E was added to the cleared lysate and mixed by pipetting. A quantity of $650 \mu \mathrm{L}$ of the mixture was applied to the DNeasy Mini Spin Column placed in a $2 \mathrm{~mL}$ collection tube which was centrifuged at $6,000 \times \mathrm{g}$ while its flow-through was discarded. The rest of the mixture was applied as aforementioned. Subsequently, the DNeasy Mini Spin Column was placed in a new $2 \mathrm{~mL}$ collection tube, and $500 \mu \mathrm{L}$ of buffer AW was added to it. The tube was centrifuged at $6,000 \mathrm{x} g$ for 1 min, and the flow-through was discarded, reusing the collection tube in the next step. A total volume of $500 \mu \mathrm{L}$ of the same buffer was used once more with centrifuging at 20,000 x g for $2 \mathrm{~min}$. The DNeasy Mini Spin Column was transferred to a $1.5 \mathrm{~mL}$ eppendrof tube and a quantity of $30 \mu \mathrm{L}$ of buffer $\mathrm{AE}$ was added twice with a separation time of at least $5 \mathrm{~min}$ between them. Before storing at $20^{\circ} \mathrm{C}$, the tube was centrifuged at full speed for $1 \mathrm{~min}$.

Estimation of DNA quantification

DNA quality and quantity was tested on $0.8 \%$ agarose gel electrophoresis using Lambda DNA as a standard. Other measurements also done for DNA concentration and purity using spectrophotometer. Final concentration of DNA was adjusted to $50 \mathrm{ng} / \mu \mathrm{l}$.

Inter Simple Sequence Repeats (ISSR) / PCR reaction mixture and program

Twenty ISSR primers (Table 1) were used for the amplification of random ISSR banding patterns according to
Table 1 List of the used ISSR primers

\begin{tabular}{cll}
\hline No. & Primer name & \multicolumn{1}{c}{ Primer sequence $\left.\left(5^{\prime} \rightarrow 3\right)^{`}\right)$} \\
\hline 1 & $\# 4$ & 5 GAG AGA GAG AGA GAG AYG3 \\
2 & $\# 811$ & GAG AGA GAG AGA GAG AC \\
3 & S-17 & GAG AGA GAG AGA GAG AT \\
5 & S-19 & GAG AGA GAG AGA GAG AA \\
6 & S-13 & ACA CAC ACA CAC ACA CYG \\
7 & S-14 & AGA GAG AGA GAG AGA GT \\
8 & S-16 & AGA GAG AGA GAG AGA GG \\
9 & S-27 & BDB CAC ACA CAC ACA CA \\
10 & S-31 & AGA GAG AGA GAG AGA GVC \\
11 & UBC-855 & ACA CAC ACA CAC ACA CYA \\
12 & $\# 890$ & VHV GTG TGT GTG TGT GT \\
13 & $\# 841$ & GAG AGA GAG AGA GAG AYC \\
14 & S-30 & HVH TGT GTG TGT GTG TG \\
15 & $\# 840$ & GAG AGA GAG AGA GAG AYT \\
16 & $\# 836$ & AGA GAG AGA GAG AGA GYA \\
17 & $\# 826$ & ACA CAC ACA CAC ACA CC \\
18. & $\# 825$ & ACA CAC ACA CAC ACA CT \\
19 & $\# 820$ & GTG TGT GTG TGT GTG TC \\
20 & $\# 818$ & CAC ACA CAC ACA CAC AG \\
\hline
\end{tabular}

Where $Y:(C, T) ; B:(C, G, T) ; D:(A, G, T) ; H:(A, T, C) V:(A, C, G)$; and $R:(A, G)$.

Sabir et al. (2009). PCR reactions were carried out in a 25 $\mu \mathrm{l}$ volume mixture containing: $5 \mu \mathrm{l}$ of a total DNA (50 ng), $4 \mu \mathrm{l}$ primer $(5 \mu \mathrm{M}), 2 \mu \mathrm{l}$ dNTPs $(200 \mathrm{mM})$ (Fermentas), $2.5 \mu \mathrm{l}$ Taq buffer $(10 \mathrm{X}), 2 \mu \mathrm{l}$ magnesium chloride $(25 \mathrm{mM})$ and $1.5 \mathrm{U}$ of Taq DNA polymerase (Hy Labs). Consequently, DNA was amplified by PCR on a Peltier Thermal Cycler200 (MJ Research. Inc, Watertown, MA) and the PCR program was: 1 cycle, $94^{\circ} \mathrm{C}(3 \mathrm{~min}) ; 40$ cycles, $94^{\circ} \mathrm{C}(1 \mathrm{~min})$, $54^{\circ} \mathrm{C}(1 \mathrm{~min}), 72(2 \mathrm{~min}) 1 \mathrm{cycle}, 72^{\circ} \mathrm{C}(7 \mathrm{~min})$, and then cooling down to $4^{\circ} \mathrm{C}$.

\section{ISSR-Gel processing}

Amplified products $(25 \mu \mathrm{l})$ were mixed with $5 \mu \mathrm{l}$ of orange gel loading buffer and analyzed by electrophoresis in 3\% agarose gels (Hy Labs) in 1X TAE buffer at 4 volt/cm for $4 \mathrm{~h}$ as well as detected by staining with ethidium bromide (Sigma). A 100 bp DNA ladder was used as standard marker (Fermentas). Consequently, amplicons were visualized and photographed black and white on Polaroid type film with UV trans-illuminator (ImageMaster $® V D S)$. DNA bands were scored (1) for presence and (0) for absence for each primer- 
genotype combination. Only reliable and clear bands were scored for the estimation of genetic similarity.

\section{ISSR Data analysis}

Data matrix was utilized to generate genetic similarity data among genotypes using. Jacquard's similarity coefficient formula as the following:

$S_{i j, \text { Jaccard }}=\frac{n_{11}}{n_{11}+n_{01}+n_{10}}$

Where $n x y$ is the number of characters that have state $x$ in individual $i$ and state $y$ in individual $j$. Un-weighted pair group method using arithmetic averages (UPGMA) (Schluter and Harris 2006) phenogram was then calculated from the Jaccard's similarity using fingerprint analysis with missing data (FAMD) software version 1.108 beta. Tree view software (Win32) version 1.6.6 was used to visualize the resulted trees. All trees presented in this study were mid-pointed.

\section{Results}

Genetic variations and relatedness among grapevine accessions based on ISSR

Among the 20 tested primers used to investigate the pattern of genetic variation between 36 accessions of grape grown at the southern region of West-Bank, Palestine; 17 primers produced reasonable amplification products with high intensity and pattern stability (Table 2), whereas, only 2 primers (S-27 and \#841) exhibited ambiguous, light, and non-clear complex amplification products and primer \#840 produce no amplification, and therefore were excluded from our analysis.

A total of 57 DNA fragments (loci) separated by electrophoresis on agarose gels, were detected (Table 2), ranging in size from 150 to 900 bp (Fig. 2). Of these fragments, $55(88 \%)$ were polymerphic and $2(3.5 \%)$ were monomorphic. Our results also revealed an average of 3.1 loci per primer (Table 2). A minimum of 1 and a maximum of 10 DNA fragments were obtained using (S-17, \#820 and \#841) and (S-31) primers, respectively (Table 2). There-

Table 2 Analysis of the polymorphism obtained with ISSR markers

\begin{tabular}{|c|c|c|c|c|c|c|c|}
\hline & & $\begin{array}{l}\text { Ap } \\
\text { band }\end{array}$ & $\begin{array}{l}\text { nate } \\
\text { (bp). }\end{array}$ & & & & \\
\hline $\begin{array}{l}\text { primers } \\
\text { name }\end{array}$ & $\begin{array}{c}\text { Total No. ISSR } \\
\text { bands }\end{array}$ & Min. & Max. & $\begin{array}{c}\text { Mono-morphic } \\
\text { band }\end{array}$ & $\begin{array}{l}\text { Poly-morphic } \\
\text { bands }\end{array}$ & $\begin{array}{c}\text { Poly-morphic } \\
(\%)\end{array}$ & Primer case \\
\hline$\# 9$ & 6 & 200 & 800 & 0 & 6 & $100 \%$ & Included \\
\hline S-13 & 2 & 300 & 400 & 0 & 2 & $100 \%$ & Included \\
\hline$\# 4$ & 5 & 150 & 400 & 0 & 5 & $100 \%$ & Included \\
\hline S-17 & 1 & 400 & 400 & 0 & 1 & $100 \%$ & Included \\
\hline S-19 & 3 & 250 & 600 & 0 & 3 & $100 \%$ & Included \\
\hline S-14 & 3 & 250 & 500 & 0 & 3 & $100 \%$ & Included \\
\hline S-16 & 5 & 150 & 400 & 0 & 5 & $100 \%$ & Included \\
\hline S-27 & 4 & 250 & 450 & 4 & 0 & $0 \%$ & Excluded \\
\hline$S-31$ & 10 & 180 & 800 & 2 & 8 & $80 \%$ & Included \\
\hline \# 818 & 5 & 250 & 700 & 0 & 5 & $100 \%$ & Included \\
\hline \# 820 & 1 & 300 & 300 & 0 & 1 & $100 \%$ & Included \\
\hline$\# 825$ & 2 & 300 & 400 & 0 & 2 & $100 \%$ & Included \\
\hline \# 826 & 2 & 400 & 450 & 0 & 2 & $100 \%$ & Included \\
\hline \#836 & 2 & 600 & 700 & 0 & 2 & $100 \%$ & Included \\
\hline S-30 & 2 & 300 & 400 & 0 & 2 & $100 \%$ & Included \\
\hline \#841 & 1 & 350 & 350 & 1 & 0 & $0 \%$ & Excluded \\
\hline \#890 & 2 & 300 & 400 & 0 & 2 & $100 \%$ & Included \\
\hline UBC-855 & 1 & 300 & 300 & 0 & 1 & $100 \%$ & Included \\
\hline \# 811 & 5 & 280 & 900 & 0 & 5 & $100 \%$ & Included \\
\hline Total & 62 & & & 7 & 55 & $88 \%$ & \\
\hline
\end{tabular}




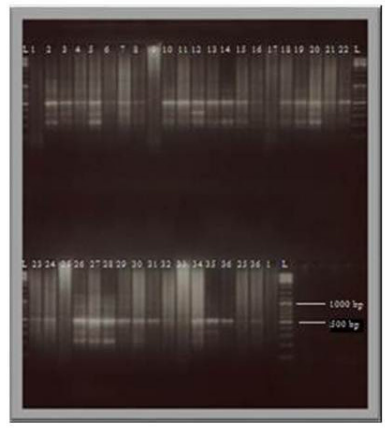

S-14

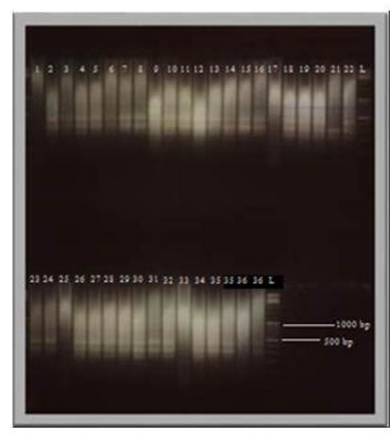

S-13

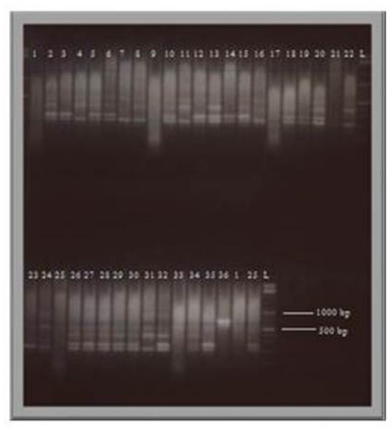

\#9

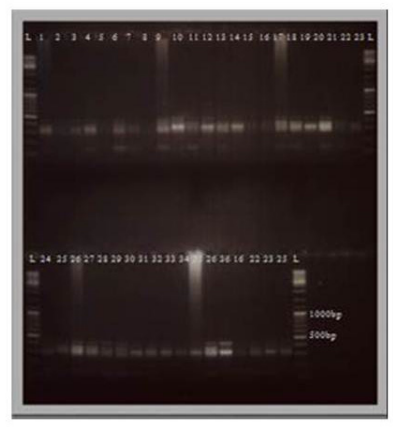

S-16
Fig. 2 Examples of ISSR banding patterns generated in Palestinian Grapevine cultivars using S-14, \#9, S-13, and S-16 primers (100 bp ladder)

fore, the later primer (S-31) is considered as the most powerful primer among the tested once (Fig. 2). The maximum percentage of polymorphic markers was $100 \%$ with 17 primers (Table 2), however, the minimum percentage was $80 \%$ with only one primer (S-31).

\section{Genetic distances}

The data matrix size analyzed included 2232 entries, 1288 of which were for present loci (1) and 944 for absent loci (0). Accordingly, the Jaccard coefficient was calculated and presented in table 7. The genetic distance matrix showed an average distance range from 0.05 to 0.76 with a mean of 0.405 . The maximum genetic distance value of 0.76 (24\% similarity) was exhibited between (Shami and Marawi. Hamadani.Adi) as well as (Bairuti and Marawi.Hamadani.Adi) genotypes, whereas the lowest genetic distance of 0.05 (95\% similarity) was exhibited between (Jandali.Tawel.Mofarad and Jandali.Kurawi.Mlzlz) along with (Shami.Aswad and Shami.mtartash.mlwn) genotypes.

UPGMA analysis

UPGMA dendrogram clustered the grape genotypes into eight major clusters in addition to an isolated genotype

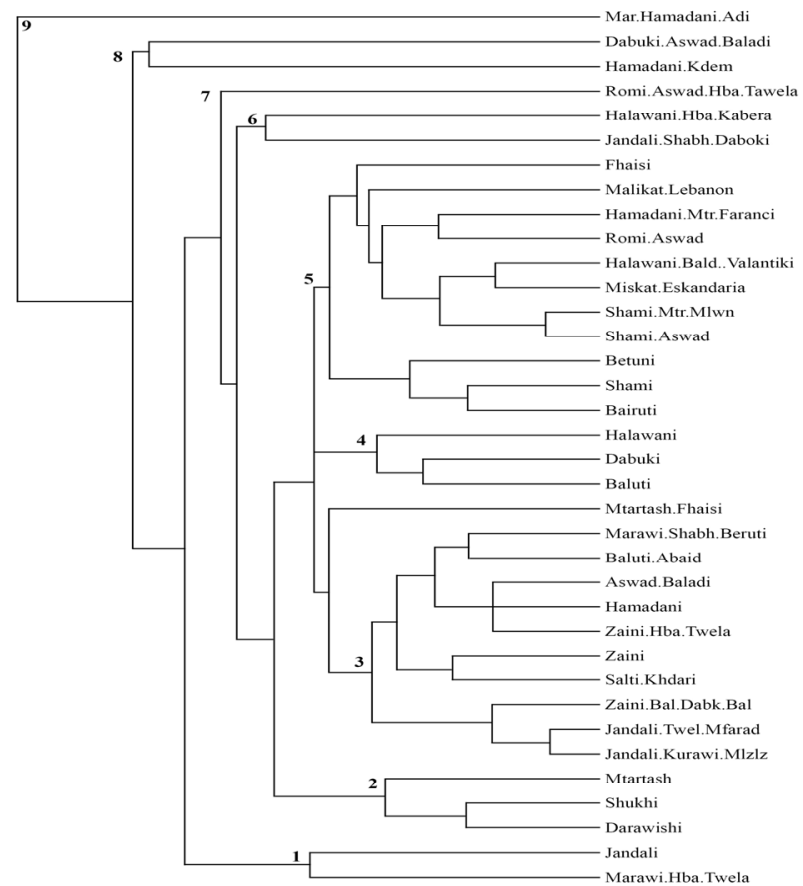

Fig. 3 Dendrogram of 36 local Palestinian Grapes constructed by UPGMA based on ISSR banding patterns

Marawi.Hamadani.Adi as a distinctive one (Fig. 3). Cluster "1", consists of only two genotypes: Marawi.Habe.Tawela and Jandali. Cluster "2" contains Darawishi and Shukhi genotypes which both are related to Mtartash. Cluster "3" is further divided into two highly related small groups (Jandali.Kurawi.Mlzlz, Jandali.Tawel.Mafrod, and Zaini.Baladi. Dabuki.Baladi) and (Sulti.Khdari, Zaini, Zaini.Habe.Tawela, Hamadani, Aswad.Baladi, Baluti.Abiad and Marawi.Shabh. Bairuti), in which all also are connected into Mtartash. Fhaisi cultivar. Cluster "4" composed of (Baluti and Dabuki) related to Halawani.

Cluster "5" is sub-divided into small groups including ("Bairuti and Shami" related to Betuni), (highly related Shami.Aswad and Shami.Mtartash.Mlwn), (Miskat.El. Eskandaria and Halawani.Baladi.Valantiki), (Romi.Aswad and Hamadani.Ma'tar.Faranci), and another two related individual genotypes Malikt.Libnan and Fhaisi.

Cluster "6" consists of Jandali.Shabh.Dabuki and Halawani. Habe.Kabera. Cluster " 7 " composed of only one genotype Romi.Aswad.Habe.Tawela. Finally, cluster "8" consists of Hamadani.Kadem and Dabuki.Aswad.Baladi.

\section{Discussion}

Grape (Vitis vinifera L.) is known for its wide morphological and genetic diversity and the existence of large 
Table 3 Jaccard's distance index generated for the 36 local Palestinian grapevines' ISSR data

\begin{tabular}{|c|c|c|c|c|c|c|c|c|c|c|c|c|c|c|c|c|c|c|c|c|c|c|c|c|c|c|c|c|c|c|c|c|c|c|c|c|c|c|}
\hline 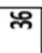 & & & & & & & & & & & & & & & & & & & & & & & & & & & & & & & & & & & & & 8 & 定 \\
\hline$\ddot{m}$ & & & & & & & & & & & & & & & & & & & & & & & & & & & & & & & & & & & & 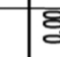 & 영 & 蛋 \\
\hline क & & & & & & & & & & & & & & & & & & & & & & & & & & & & & & & & & & & & 8 & 㝵 & 凬 \\
\hline $\mathscr{m}$ & & & & & & & & & & & & & & & & & & & & & & & & & & & & & & & & & & & & 府: & 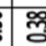 & of \\
\hline$\widetilde{m}$ & & & & & & & & & & & & & & & & & & & & & & & & & & & & & & & & & & $8:$ & 영 & 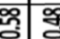 & 害 & gog \\
\hline$\vec{m}$ & & & & & & & & & & & & & & & & & & & & & & & & & & & & & & & & & ষ্ণচ: & 商 & $\overrightarrow{8}$ & 危: & लू & 管 \\
\hline p) & & & & & & & & & & & & & & & & & & & & & & & & & & & & & & & & 8 & 高: & 总 & F & 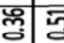 & 戹 & $\frac{0}{6}$ \\
\hline 9 & & & & & & & & & & & & & & & & & & & & & & & & & & & & & & & 8 & 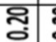 & ¿্ণ & ס: & 要 & 屃: & รั & : \\
\hline$\Phi$ & & & & & & & & & & & & & & & & & & & & & & & & & & & & & & ৪. & 응 & 영 & ¿্ঠীড & প্রু & 영 & 危 & $\bar{z}$ & (3) \\
\hline$\approx$ & & & & & & & & & & & & & & & & & & & & & & & & & & & & & 8 & శ్రి & 픙 & శ్రి & প্ল্রী: & 명 & శ్ర్ర & 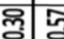 & 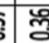 & 范 \\
\hline 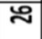 & & & & & & & & & & & & & & & & & & & & & & & & & & & & | & 녕 & 政 & 잉 & ल্口ু & ল্লুচ⿵ & 范 & శ్శ్రి & 荡 & 8 & 要 \\
\hline 2 & & & & & & & & & & & & & & & & & & & & & & & & & & & 8 & : & 咅 & శ్రి & 응 & Z्ञ & ב్రీ & 换 & ఫ్ర & 商: & 产 & סू \\
\hline 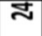 & & & & & & & & & & & & & & & & & & & & & & & & & & 8 & হ్ల్ర & \begin{tabular}{|l|}
$\%$ \\
\end{tabular} & $\approx$ & 产 & & ర్ల్ర & : & $\overline{0}$ & go & 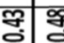 & 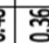 & 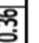 \\
\hline$\Phi$ & & & & & & & & & & & & & & & & & & & & & & & & & 8. & $\bar{m}$ & $\overrightarrow{\mathrm{m}}$ & 预 & 前 & 蛋 & 戹 & 疋 & శ్ల్ర & s: & চ్ & 8 & $z$ & 超 \\
\hline$\pi$ & & & & & & & & & & & & & & & & & & & & & & & & 8 & 공 & 必 & $\mathscr{丷}$ & $\mid \%$ & $\mid \frac{\pi}{6}$ & 悉 & శ్రి & $\stackrel{\infty}{6}$ & প্ষ্ঠিত & 楁 & 要 & 商: & 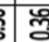 & 范 \\
\hline$\vec{z}$ & & & & & & & & & & & & & & & & & & & & & & & 8 & శ్రి & శ్రి & 必 & 9 & 朵 & 孞 & : & 영 & 켱 & ఫ্ঠী & 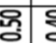 & 题 & 용| & ল్ల్ & ת. \\
\hline న్ & & & & & & & & & & & & & & & & & & & & & & ర్రి & $\approx$ & శ్రి & $\overrightarrow{\text { \% }}$ & $\overline{\tilde{g}}$ & $\vec{\rho}$ & 吅 & 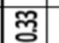 & চ্ণু & \%్ర్య & స్ & 厕 & : & 启 & : & 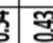 & 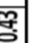 \\
\hline 9 & & & & & & & & & & & & & & & & & & & & & 8 & 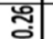 & 咅 & శ్ల్య & 产 & శ్ల్ర & శ్రి & $\mid \approx$ & 疋 & శ్ర & $\mathbb{\sigma}$ & ఫ్రి & 志: & ס: & 항 & 产 & $\bar{g}$ & 商 \\
\hline$\stackrel{29}{a}$ & & & & & & & & & & & & & & & & & & & & 8 & $\bar{\delta}$ & 2్రి & \%్্ু⿹ & F & F & 产 & প্তু & $\overrightarrow{|c|}$ & $\overrightarrow{\mathrm{g}}$ & ల్ల్ర & लㅇㅇㅇ & ब্ & 䨱? & 8 & & 梀 & 8 & 8 \\
\hline 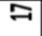 & & & & & & & & & & & & & & & & & & & 8 & 응 & 공 & প্তি & : & 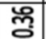 & F & 递 & ్ㅕㅇ & 兑 & 䨱 & 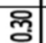 & \%్ర్ & \%্ণ & $\vec{\delta}$ & $\overrightarrow{\text { जु }}$ & F & 왕 & $\bar{\Xi}$ & F \\
\hline$\stackrel{9}{9}$ & & & & & & & & & & & & & & & & & है & & gi: & 夏 & $\overline{\tilde{g}}$ & প্তি & প্পু & : & 敢 & 명 & 荡 & 条 & 商 & ल्ले & $\overline{\hat{g}}$ & స్ర & $\vec{\delta}$ & 항 & 영 & 危 & 5 & 붕 \\
\hline$\stackrel{2}{2}$ & & & & & & & & & & & & & & & & हू & 8 & 愙 & 前 & 产 & ஜ্ণ & প্তি & \%ั & $\widetilde{\tilde{g}}$ & \% & 영 & $\overrightarrow{\mathrm{g}}$ & 茼 & 응 & চ্ণী & \%్g & శ్ర్య & 㴦 & 항 & F & 产 & $\overline{8}$ & $\overrightarrow{8}$ \\
\hline $\overrightarrow{7}$ & & & & & & & & & & & & & & & 8 & ডِ & 官 & స్ & 蓉/2 & శ్ర్ర & Z्. & 2్రి & 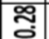 & ס্ণি & \% & $\approx$ & జ్ర్ర & 敢 & \%্ণি & 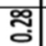 & శ్ల్ర & ఫ్ర్ర & ত্ণী: & ్ㅐㅇ & 앙 & F्: & 8 & 害 \\
\hline 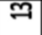 & & & & & & & & & & & & & & 8 & $\stackrel{\infty}{2}$ & $\tilde{z}$ & ত্ডিন & 容 & శ్రిల & প్্চি & 응 & $\overrightarrow{\mathrm{g}}$ & శ్రి & 2: & \%్ㅇㅇ & 要 & : & হ্লি & 220 & ఫ్రి & శ్ర & స్ర & శ్రిక & हु) & F & g: & $\bar{z}$ & 㞼 \\
\hline$\approx$ & & & & & & & & & & & & & 8 & 戹 & శొ & $\tilde{m}$ & $\frac{0}{3}$ & 范 & ন্ঠি: & శ్రి & $\ddot{z}$ & శ్రి| & প্gু & 商 & F & \% & প্তি & 弚 & $\overrightarrow{\mid \vec{g}}$ & ల్ల్ర & 垎 & ల్ల్ర & 戹 & 영 & 양 & 영 & $\exists$ & 砉 \\
\hline$\exists$ & & & & & & & & & & & & 8 & శ్రి & $\approx$ & ल & 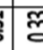 & 售 & m్g & శ్రి & 守 & శ్రి & శ్రి & : & \%్రి & 卷 & \% & 䒿 & 飠 & 欮 & ল্পু & శ్రి & ल্ֶু & 戹 & $\overline{8}$ & 웡 & : & go & 害 \\
\hline 으 & & & & & & & & & & & 8 & శ্ণী & İ & প্ত্তি & $\approx$ & $\stackrel{\sim}{2}$ & 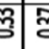 & $\overline{\mathrm{c}} \overline{\mathrm{c}}$ & $\bar{\delta}$ & శ్ర్రి & 긍 & ت्ञ & 㕚 & 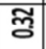 & 青 & శ్్ㅇ & శ్రా & $\approx \widetilde{g}$ & 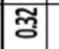 & ल्্ुু & ल্gুo & চ্ণি & 商: & $\overrightarrow{\text { ज्ञ }}$ & 응 & 曲 & $\bar{E}$ & 宫 \\
\hline or & & & & & & & & & & 8 & \% & \begin{tabular}{|l|}
$g$ \\
\end{tabular} & g & 8 & $g$ & 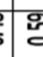 & 8 & 8 & F्: & శ్ల్ర & छ & 융 & F & 웜 & 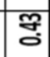 & $\vec{g}$ & Z & \begin{tabular}{|l|} 
\\
\end{tabular} & \begin{tabular}{|l|} 
\\
\end{tabular} & : & 商 & 马্ & 宅: & 망 & 喿 & 害 & $\bar{g}$ & 客 \\
\hline$\infty$ & & & & & & & & & 8 & 营 & శ్రి & 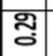 & ल্gুᄋ & 厚 & $\Xi$ & $\bar{\Sigma}$ & 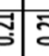 & $\vec{\delta}$ & 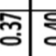 & 导 & ल্ণু & 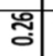 & $\stackrel{\square}{\square}$ & 总 & 앙 & 类 & 융 & \begin{tabular}{|l|} 
gog \\
\end{tabular} & 桊 & ले & 总 & ন্ডি & ¿্రత) & 항 & 炙 & g्वी & 3 & 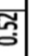 \\
\hline 7 & & & & & & & & 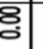 & స్రి & 寻 & జ్రి & 夋 & F్ & 厌 & \% & (ֶ) & 2 & $\overline{\widetilde{m}}$ & $\overline{\tilde{\sigma}}$ & 总 & $\underset{\Xi}{\infty}$ & Zే: & $\tilde{\Xi}$ & 总 & શ્త & 总 & : & 융 & 䒺 & 前 & 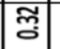 & : & 网 & 商 & 응 & 产 & gु & $\frac{8}{8}$ \\
\hline 6 & & & & & & & 8 & శี & : & 周 & \%్ర̆ & $\bar{y}$ & $\bar{y}$ & $\vec{~}$ & ল্ল & (ֶ) & $8=$ & 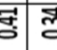 & 菏 & ল্লg & $\bar{\sigma}$ & శ్ర & $\bar{\sigma}$ & శ్రి & $\overrightarrow{\mathrm{g}}$ & ল্লğ & ż & ল্লু & 鬲 & প্তি & 융 & స్ర & 芯 & $\overrightarrow{\text { जु }}$ & 응 & 영 & $\bar{z}$ & $\overline{8}$ \\
\hline$\pi$ & & & & & है & Ș & 禺 & శ్రి & $\vec{\sigma}$ & 웅 & \%응 & శ్రి & శ్రి & 巫 & 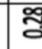 & co & (2) & 응 & శ్రిశ & ल্লু & ఫ্ণ & প্তি & \%్ & \%оㅇㅇ & F & F & శ్ర & 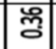 & 束 & 의 & $\bar{y}$ & 胥 & ఫ્ડ & 항 & 응 & 영 & $g$ & g \\
\hline$\sigma$ & & & & ह & 3 & & 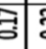 & శ్ & 范 & $\widetilde{\widetilde{g}}$ & $\dddot{2}$ & ż & \%్ & 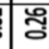 & $\bar{\Xi}$ & $\stackrel{\tilde{c}}{\approx}$ & $\overline{2}$ & & 䛎 & $\widetilde{\widetilde{\gamma}}$ & $\vec{\sigma}$ & 링 & $\vec{g}$ & $\vec{g}$ & ল্লু & $\vec{g}$ & శ్ర & $\overrightarrow{\mathrm{g}}$ & $\vec{g}$ & ฉ્d & 경 & $\approx$ & ন্ঠি & 맹 & 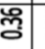 & 商 & $\stackrel{2}{2}$ & के \\
\hline$m$ & & & 8 & $\bar{~}$ & 30 & & 官 & 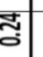 & ః્ర & gి & 킁 & ஜ్ల్ & శ్రి & 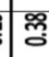 & लू & $\bar{\pi}$ & 药 & 敢 & চ্ঠিণ & ळ్ల్ర & ટ્ర & శ్ర & চ্ণি & 冎 & $\mathbb{F}$ & $\overline{\mathrm{g}}$ & $\vec{\delta}$ & 웡 & 票 & o & $\mid \frac{m}{\delta}$ & ్ㅏㅇ & $\widetilde{్}$ & B & 商 & के & : & 串 \\
\hline & & 8 & $\stackrel{\square}{\circ}$ & 10 & $c$ & 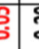 & 兰 & $\bar{\delta}$ & స్తి & के & జ్రి & হ్ల్g & \%్ర & ल্ & $\overline{\bar{c}}$ & 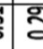 & ম্ডেন & m. & হ్্ֶু & 产 & న్రి & হ্ল్ర & 递 & 品 & F & F & প্লুণ & 商 & \begin{tabular}{|l|} 
of \\
\end{tabular} & 昰 & 炙 & হ্লু & ন্ড: & छ्ठ & 융 & 今్ & $\overline{3}$ & $\overrightarrow{3}$ \\
\hline 7 & 8 & 8 & $\bar{\theta}$ & 总 & 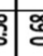 & & 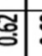 & 8 & लू & $\vec{n}$ & $\approx$ & 安 & 吾 & 녕 & : & 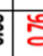 & 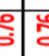 & $\frac{0}{0}$ & 8 & 융 & $\mathscr{8}$ & 형 & 영 & 동 & 병 & 영 & ஜ: & :्ठ্ট: & $\ddot{~}$ & : & : & ㅂㅇㅇ & छ্: & 8 & 롱 & 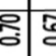 & 5 & 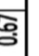 \\
\hline & 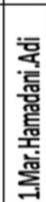 & 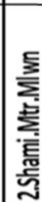 & 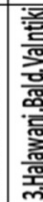 & 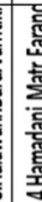 & 言 & 产 & 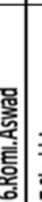 & 音 & 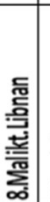 & $\begin{array}{l}: \overline{\overline{\mathrm{m}}} \\
\frac{\mathrm{m}}{\mathrm{m}}\end{array}$ & 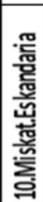 & 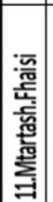 & 离 & 동 & 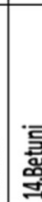 & 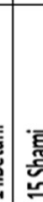 & 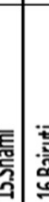 & 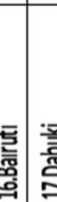 & 륻 & $\begin{array}{l}\text { 劲 } \\
\text { 总 }\end{array}$ & 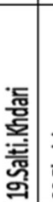 & $\begin{array}{l}: \frac{5}{\bar{n}} \\
\text { : } \\
\text { : }\end{array}$ & 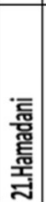 & & 畜 & 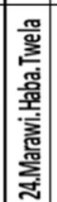 & 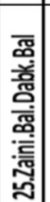 & 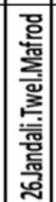 & 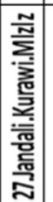 & 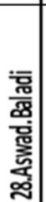 & 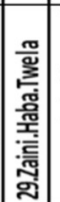 & 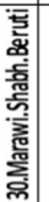 & 总 & 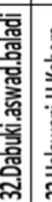 & 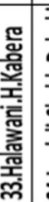 & 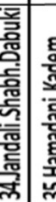 & 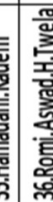 & 产 \\
\hline
\end{tabular}


number of cultivars (Santos et al. 2013). Therefore, determination of genetic variability and proper cultivar identification would be of major importance for any future breeding programs and germplasm collection (Jing et al. 2013). In the present study, the level of polymorphism among 36 grapevine (Vitis venifera L.) accessions commonly grown in Palestine were estimated using DNA-based marker technique, in which 20 ISSR primers (dinucleotide repeats, commonly used for grape identification; Wang et al. 1994; Moreno et al. 1998), were used to identify and discriminate the Palestinian grapes. Many authors stated that, few ISSR primers (5 primers) were needed to generate diagnostic and reproducible fingerprint profiles and therefore distinguishing between the varieties (Moreno et al. 1998).

Of these primers screened initially on 36 grapevine cultivars, 18 primers yielded an altered interval of polymorphism from 1 to 8 . However, the other two primers (S-27 and \#841) were excluded from the analysis since they produced no polymorphism. Reddy et al. (2002) and Herrera et al. (2002) reported that some primers were more efficient in recognizing a complementary site in the plant genome. Comparing with Herrera and others (2002), our results revealed higher polymorphic bands (55 bands) using 18 primers relating to 40 bands using 11 primers (which are parallel to our examined primers). Interestingly, primer \#820 that showed only one band in Merlot cultivar cultivated in Chile also revealed the same number of bands (1 band) with our Bairuti genotype so these genotypes maybe related, however, further investigation is needed to support this assumption.

Among the evaluated 20 primers; 6 were GA repeats (the maximum), 5 were $\mathrm{AC}$ repeats, 4 were $\mathrm{AG}$ repeats, 3 were GT repeats, and 2 were CA repeats. Remarkably, 3 motifs (AG) of nucleotide repeats were realized, with $100 \%$ polymorphism (Table 3 ). Consequently, the primers revealed significant differences in resolving polymorphism, in which the primers containing (GT)n repeats and (CA)n repeats were the most polymorphic. The same is true for the average number of bands per primer.

Thomas and others (1993) found the repeats GA and GT as the most highly represented in the Vitis genome. Further, Dhanorkar and others (2005) recorded the repeats $\mathrm{AC}$ as more common among ISSR primers selected by using Indian grapes in a more recent study.

Contradictory to the fact that AT motifs in the plant kingdom have generally been approved as the most plentiful repeat (Casasoli et al. 2001), here, in grapes our results revealed $4 \mathrm{AG}$ motifs among the 20 tested primers which confirm the results of Dhanorkar et al. (2005) who also registered 6 AG motifs in Indian grapes. Consequently, the relative abundance of nucleotide repeats in the grapevine genome indicates differences between different studies conducted on Vitis species.

The size of amplified fragments varied from $150 \mathrm{bp}$ to $900 \mathrm{bp}$. This interval was narrower than the results obtained by Dhanorkar, and others (2005) and Sabir and others (2009), who reported fragments between (300 bp and 1500 bp) and (300 bp and $2500 \mathrm{bp}$ ), for different grape cultivars in India and Turkey, respectively. Indeed, the narrower amplified fragments exhibited by our cultivars might related to the smaller and restricted cultivated areas (Hebron and Bethlehem regions, about $2000 \mathrm{Km}^{2}$ ) in which our grapevines are grown comparing with the very large studied areas of India and Turkey.

The percentage of polymorphism revealed in the present analysis is much higher than those reported by Moreno et al. (1998); Dhanorkar et al. (2005); Sabir et al. (2009). This could be attributed to the pre-selection of our primers for their abilities to generate clear and polymorphic-bandpatterns and/or the intra-varietal differences as it is presented in our study rather than the inter-varietal differences among the examined cultivars.

The values of the genetic distances ranged from 0.05 for the most closely related cultivars (Jandali.Tawel.Mofarad and Jandali.Kurawi.Mlzlz) and (Shami.Mtaratsh.Mlwn and Shami.Aswad) to 0.76 for the most distantly related cultivars (Marawi.Hamadani.Adi and Shami) and (Marawi.Hamadani. Adi and Bairuti). These results confirm that these cultivars could be the same cultivar but with different names (synonyms). Vignani (1996) set a precedent that closely related individuals could be considered under a common name. UPGMA dendrogram (Fig. 3) clustered the grape genotypes into eight major clusters including 2, 3, 11, 3, 11, 2, 1 and 2 genotypes, respectively. In addition to that, cluster 9 (Marawi.Hamadani.Adi) was isolated as a distinctive genotype. Interestingly, some examined cultivars showed high similarities with each other such as (Jandali.Tawel.mafrod and Jandali.Kurawi.Mlzlz, by 95\%; Shami.Aswad and Shami. Mtartash.Mlwn, by also 95\%), which might assumed that these cultivars are highly correlated and therefore might be the same cultivars with different names. The most distant cultivars were between (Marawi.Hamadani.Adi and Shami, by $24 \%$ ) and (Marawi.Hamadini.Adi and Bairuti, by $24 \%$ ). Therefore, these cultivars could be useful and interesting grape genotypes for any future breeding program in Palestine. In addition, since ISSR represents an efficient tool for estimating the genetic variability and the genetic relationships among our examined grapevine genotypes therefore, ISSR 
markers could be a useful technique for grapevine genotype identification's and characterizations.

\section{References}

Achtak H, Oukabli A, Ater M, Santoni S, Kjellberg F, Khadari B (2009) Microsatellite Markers as Reliable Tools for Fig Cultivar Identification. J Amer Soc Hort. Sci 134(6):624-631

Agarwal M, Shrivastava N, Padh H (2008) Advances in molecular marker techniques and their applications in plant sciences. Plant Cell Reports 27:617-631

Bahurupe J, Sakhare S, Kulwal P, Akhare A, Pawar B (2013) Genetic diversity analysis in chilli (Capsicum anmum L.) using RAPD markers. The international quarterly journal of life sciences 8(3):915-918

Basheer-Salimia R, Awad M, Ward J (2012). Assessments of Biodiversity Based on Molecular Markers and Morphological Traits among West-Bank, Palestine Fig Genotypes (Ficus carica L.). American Journal of Plant Sciences 3(9):1241-1251

Basheer-Salimia R (2015a) Ampelographic and phenotypic characterizations of white Palestinian grapevine cultivars. Palestine Technical University-Kadoorie Research Journal 3(1):1-11

Basheer-Salimia R (2015b) Identification of Palestinian coloredtable-grape cultivars by means of morphological and pomological descriptors. American Journal of Experimental Agriculture 9 (5): $1-15$

Basheer-Salimia R, Ward J (2014) Climate change and its effects on olive tree physiology in Palestine. Review of Research Journal 3(7):1-7

Casasoli M, Mattioni C, Cherubini M, Villani F (2001) Genetic linkage map of European chestnut (Castenea sativa Mill.) based on RAPD, ISSR and isozyme markers. Theor Appl Genet 102:1190-1199

Chittora M, Sharma D, Veer C, Verma G (2015) Molecular markers: An important tool to assess genetic fidelity in tissue culture grown long-term cultures of economically important fruit plants. Asian Journal of Bio Science 10(1):101-105

Choudhary RS, Zagade_Maboodurrahman VS, Khalakar GD, Singh NK (2014) ISSR based genotypic differentiation of grape (Vitis vinifera L.) Bioscan 9(2):823-828

Depnath S (2009) Development of ISSR markers for genetic diversity studies in Vaccinium angustifolium. Nordic Journal of Botany 27:141-148

Dhanorkar VM, Tamhankar SA, Patil SG, Rao VS (2005) ISSR-PCR for assessment of genetic relationships among grape varieties cultivated in India. Vitis 44 (3): 127-131

Herrera R, Cares V, Wilkınson MJ, Caligari PD(2002) Characterization of genetic variation between (Vitis vinifera) cultivars from central Chile using RAPD and inter simple sequence repeat markers. Euphytica 124:139-145
Jing Z, Wang X, Cheng J (2013) Analysis of genetic diversity among Chinese wild Vitis species revealed with SSR and SRAP markers. Genet Mol Res 12(2):1962-1973

Lima ML, Garcia AA, Oliveira KM, Matsuoka S, Arizono H, De Souza-CL Jr, De-Souza AP (2002) Analysis of genetic similarity detected by AFLP and coefficient of parentage among genotypes of sugar cane (Saccharum spp.). Theor Appl Genet. 104(1): 8-30

MOA (2017) Strategy for grape sector in Palestine "2017-2022". Publications of the Ministry of Agriculture, Ramallah, Palestine

Moreno S, Martin JP, Ortiz JM (1998) Inter-simple sequence repeats PCR for characterization of closely related grape vine germplasm. Euphytica 101:117-125

Qian W, Ge S, Hong DY (2001) Genetic variation within and among populations of a wild rice Oryza granulata from China detected by RAPD and ISSR. Theor Appl Genet 102:440-449

Reddy M, Sarla N, Siddiq EA (2002) Inter simple sequence repeat (ISSR) polymorphism and its application in plant breeding. Euphytica 128:9-17

Riaz S, Gabriella De-Lorenzis G, Velasco D, Koehmstedt A, Maghradze D, Bobokashvili Z, Musayev M, Zdunic G, Laucou V, Walker M, Failla O, Preece J, Aradhya M, Arroyo-Garcia R (2018) Genetic diversity analysis of cultivated and wild grapevine (Vitis vinifera L.) accessions around the Mediterranean basin and Central Asia. BMC Plant Biology 18 (137):1-14

Sabir A, Tangolar S, Buyukalaca S, Kafkas S (2009) Ampelographic and molecular diversity among grapevine (Vitis spp.) cultivars. Czech Journal of Genetic and Plant Breeding 45(4):160-168

Santos AR, Duarte D, Sousa S, Eiras_Dias J, Gomes AC (2013) An optimized set of microsatellites for grapevine identification. $36^{\text {th }}$ world congress of vine and wine; 2-7 June 2013 Bucharest, Romania (Organisation Internationale de la Vigne et du Vin: Paris, France)

Schluter PM, Harris SA(2006) Analysis of multi-locus fingerprinting data sets containing missing data. Mol Ecol 6:569-572

Sripholtaen A, Charoenchai C, Urairong H (2016). Application of microsatellite markers for identification of wine grape varieties in Thailand. Asia-Pac J Sci Technol 21(1):97-110

Sultan S (2005). Grapevines, establishing, planting, training, pruning, servicing, p. 459-464 (In Arabic)

Thomas MR, Matsumoto S, Cain P, Scott NS (1993) Repetitive DNA of grapevine: classes present and sequences suitable for cultivar identification. Theor Appl Genet 86:73-180

Vignani R, Bowers JE, Meredith CP (1996) Microsatellite DNA polymorphism analysis of clones of Vitis vinifera Sangiovese. Sci Hort 65:163-169

Wang Z, Weber JL, Zhong G, Tanksley SD (1994) Survey of plant short tandem DNA repeats. Theor Appl Genet 88:1-6

Weising K, Atkinson RG, Gardner RC (1995) Genomic finger printing by microsatellite primed PCR: A critical evaluation. PCR Meth Appl 4:249-255 